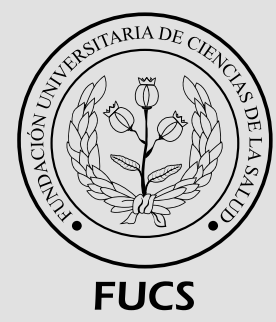

\title{
Impacto del suministro de vacunas contra COVID-19 sobre la letalidad por sars-cov-2 en colombia
}

\section{Impact of COVID-19 vaccine administration on sars-cov-2 death rate in Colombia}

a Ingeniero. Magister en Gestión de la Tecnología Educativa, Especialista en Administración de la Informática Educativa.

Docente de matemáticas e Investigador, Secretaría de Educación de Soacha, Cundinamarca.

\section{R E S U M EN}

Introducción: desde el brote inicial en Wuhan, China a finales de 2019, el SARS-CoV-2 se ha convertido en una pandemia mundial con más de 138 millones de infecciones y más de 3 millones de muertes, lo que repercute gravemente en la salud mental y la economía global. Objetivo: mostrar si hay alguna correlación de la tasa de letalidad por COVID-19 y la vacunación contra el SARS-COV-2 entre el periodo comprendido entre 17 de febrero a 4 de junio de 2021. Metodología: el trabajo de investigación se desarrolló mediante un tipo experimental, la información se obtuvo de la página web del Ministerio de Salud y Protección Social, de los informes diarios de fallecidos y vacunación contra el COVID-19 entre el periodo comprendido entre 17 de febrero a 4 de junio 2021. Resultados: el p-valor de las pruebas, para las variables tasa de letalidad y vacunación para COVID-19 es de 0,001, es menor a $\alpha=0.01$, de esta manera se acepta Ha, es decir la prueba de correlación de Pearson nos indica que existe relación entre el número de la tasa de letalidad por COVID-19 y la vacunación contra el SARS-COV-2 en Colombia. Conclusión: se considera de vital importancia establecer la ampliación de la cobertura a un $100 \%$ para los grupos de riesgo, la inclusión de estos nuevos grupos objetivos en conjunto con la ampliación de la cobertura de vacunación, lo cual hace necesaria la adquisición de nuevas dosis adicionales para combatir el SARS-COV-2 en Colombia.

Palabras clave: correlación, Pearson, COVID-19, SARS-COV-2, pandemia, vacunas, letalidad.

(C) 2021 Fundación Universitaria de Ciencias de la Salud - FUCS Este es un artículo Open Access bajo la licencia CC BY-NC-ND (http://creativecommons.org/licenses/by-nc-nd/4.0/).

\section{INFORMACIÓN DEL ARTÍCULO}

Historia del artículo:

Fecha recibido: junio 16 de 2021 Fecha aceptado: junio 28 de 2021

\footnotetext{
Autor para correspondencia.

Jorge Enrique Díaz Pinzón jediazp@unal.edu.co
}

DOI

10.31260/RepertMedCir.01217372.1237 
Introduction: since the initial outbreak in Wuhan, China in late 2019, SARS-CoV-2 has become a global pandemic accounting for over 138 million deaths, severely impacting mental health and global economy. Objective: to show if there is any correlation between the COVID-19 death rate and SARS-COV-2 vaccination in the February 17 to June 42021 period. Methodology: an experimental research design was used. Data was obtained from the Ministry of Health and Social Protection website, including the deaths and COVID-19 vaccination daily report between February 17 and June 4 2021. Results: the $\mathrm{p}$-value obtained in the tests for COVID-19 death rate and vaccination against SARS-COV-2 variables is 0.001, which is less than $\alpha=0.01$, therefore, the hypothesis (Ha) is accepted, which means the Pearson correlation test indicates there is a relation between COVID-19 death rate and SARS-COV-2 vaccination in Colombia. Conclusion: we consider it essential to achieve $100 \%$ coverage of the at-risk groups. The inclusion of this new target groups together with the expansion of vaccination coverage, makes it necessary to acquire new additional doses to combat SARS-COV-2 in Colombia.

Key words: correlation, Pearson, COVID-19, SARS-COV-2, pandemic, vaccines, lethality.

(C) 2021 Fundación Universitaria de Ciencias de la Salud - FUCS This is an open access article under the CC BY-NC-ND license (http://creativecommons.org/licenses/by-nc-nd/4.0/).

IN T RODUCCIÓN

Desde el brote inicial en Wuhan, China, a finales de $2019^{1,2}$ el SARS-CoV-2 se ha convertido en una pandemia mundial con más de 138 millones de infecciones y más de 3 millones de muertes, lo que repercute gravemente en la salud mental $^{3,4}$ y la economía global. ${ }^{5}$ En respuesta, la comunidad científica ha logrado un progreso sin precedentes, lo que ha dado como resultado la generación de múltiples vacunas, utilizando una variedad de enfoques diferentes. ${ }^{6-8}$

Después de que se secuenció genoma del coronavirus 2 del síndrome respiratorio agudo severo (SARS-CoV-2), los investigadores se aligeraron para desarrollar vacunas con el fin de frenar la propagación del COVID-19.9,10 Traspasada la inviabilidad de los bloqueos a largo plazo ${ }^{11,12}$ y al rastreo de contactos efectivo en un gran número de casos, así como la disponibilidad de varias vacunas COVID-19 admitidas, muchos países han invertido en implementaciones masivas de vacunación.

Para lograr una reducción continua de los casos de infección ${ }^{13}$ se necesitan contramedidas múltiples, incluido el distanciamiento, las pruebas y el rastreo, más aún a la luz de la reciente aparición de nuevas variantes del SARS-CoV- $2^{14}$, como B.1.1.7 y B.1.351, que según se informa tienen una mayor transmisibilidad ${ }^{15,16}$ y es probable que causen una enfermedad más grave ${ }^{17}$ en comparación con la cepa original. No se espera que la vacunación por sí sola pueda mitigar la propagación de la infección, pues es necesario coordinar una campaña de vacunación diligentemente planificada. ${ }^{18,19}$

\section{Correlación lineal de Pearson}

La cuantificación de la fuerza de la relación lineal entre dos variables cuantitativas se estudia por medio del cálculo del coeficiente de correlación de Pearson. Este coeficiente oscila entre -1 y +1 . Un valor de -1 indica una relación lineal o línea recta positiva perfecta. Una correlación próxima a cero indica que no hay relación lineal entre las dos variables. ${ }^{20} \mathrm{En}$ la tabla 1 se aprecia la escala de coeficiente de correlación de Pearson..$^{20} \mathrm{El}$ objetivo de esta investigación es manifestar si hay alguna correlación entre la tasa de letalidad por COVID-19 y la vacunación contra el SARS-COV-2, entre el periodo comprendido entre 17 de febrero a 4 de junio 2021.

Tabla 1. Escala de coeficiente de correlación de Pearson

\begin{tabular}{|l|l|}
\hline Valor & Significado \\
-1 & Correlación negativa grande y perfecta \\
$-0,9$ a $-0,99$ & Correlación negativa muy alta \\
$-0,7$ a $-0,89$ & Correlación negativa alta \\
$-0,4$ a $-0,69$ & Correlación negativa moderada \\
$-0,2$ a $-0,39$ & Correlación negativa baja \\
$-0,01$ a $-0,19$ & Correlación negativa muy baja \\
0 & Correlación nula \\
0,01 a 0,19 & Correlación positiva muy baja \\
0,2 a 0,39 & Correlación positiva baja \\
0,4 a 0,69 & Correlación positiva moderada \\
0,7 a 0,89 & Correlación positiva alta \\
0,9 a 0,99 & Correlación positiva muy alta \\
1 & Correlación positiva grande y perfecta \\
\hline
\end{tabular}

Fuente: Suárez20 


\section{TASA DE LETALIDAD}

La letalidad en sentido cabal es una proporción que expresa el número de defunciones entre el número de casos del cual estas forman parte. No obstante, universalmente, se expresa como tasa de letalidad y se registra como el porcentaje de muertes de una causa específica con relación al total de enfermos por esa causa. ${ }^{21,22}$

La letalidad es una medida de la gravedad de una enfermedad estimada desde el punto de vista poblacional y se precisa como la proporción de casos de una enfermedad que surge mortal con respecto al total de casos en un periodo determinado. La medida muestra la relevancia en términos de su disposición para producir la muerte y se deduce de la siguiente manera. ${ }^{21,22}$

$$
\text { Letalidad (\%) } \frac{\begin{array}{c}
\text { (Número de muertes por una enfermedad } \\
\text { en un periodo determinado ) }
\end{array}}{\begin{array}{c}
\text { (Número de casos diagnosticados de la } \\
\text { misma enfermedad en el mismo periodo) }
\end{array}}
$$

\section{METODOLOGÍA}

El trabajo de investigación se desarrolló mediante un tipo experimental que es el siguiente: “aquella que permite mayor seguridad al establecer relaciones de causa a efecto pues presenta una visión general y aproximada del objeto de estudio, además de contar con una investigación cuyo diseño establece un método experimental habitual del conjunto de las normas científicas". Monje (2011) citado por Díaz. ${ }^{23}$ y Shuttleworth citado por Díaz ${ }^{24,25}$ menciona que "regularmente a estos experimentos se los nombra ciencia verdadera y manejan medios matemáticos y estadísticos cotidianos para evaluar los resultados de modo concluyente. Todos los experimentos cuantitativos utilizan un formato estándar con algunas pequeñas diferencias interdisciplinarias para generar una hipótesis que será probada o desmentida. Esta hipótesis debe ser demostrable por medios matemáticos y estadísticos y constituye la base alrededor de la cual se diseña todo el experimento".

\section{POBLACIÓN}

La información se obtuvo de la página web del Ministerio de Salud y Protección Social26 y de los informes diarios de vacunación contra el COVID-19, entre el periodo comprendido entre 17 de febrero a 4 de junio 2021 .

Formulación de hipótesis: la hipótesis del presente trabajo de investigación se diseña como una relación causal y se enuncia de la siguiente forma: (a) hipótesis alterna (Ha): existe relación entre el número de la tasa de letalidad por COVID-19 y la vacunación contra el SARS-COV-2 en Colombia. (b) hipótesis nula (Ho): no existe relación entre la tasa de letalidad por COVID-19 y la vacunación contra el SARS-COV-2 en Colombia. Nivel de significancia: $=1 \%$ $=0,1$. Toma de decisión: sí el p- valor < a es aceptar Ha, entonces rechazamos la hipótesis nula y nos quedamos con la hipótesis del investigador (Ha).

\section{RESULTADOS}

En la figura 1 se aprecian los casos diarios de letalidad por SARS-COV-2 entre 1 de enero y 4 de junio 2021 y la vacunación contra COVID-19, durante el período 17 de febrero 2021 hasta 4 de junio 2021. Se describe en ella una diferencia en la tendencia de las líneas de vacunas (azul) y la letalidad (naranja). Es decir, mientras aumenta el número de vacunas en el tiempo, disminuye la tasa de letalidad.

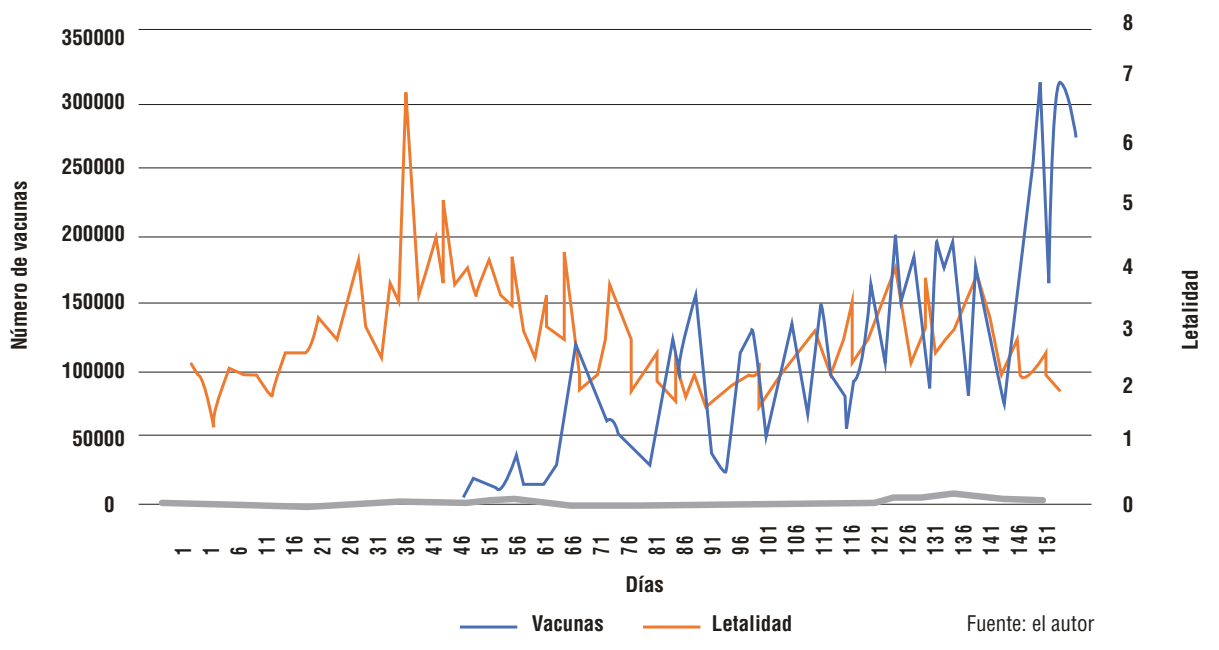

Figura 1. Vacunación y tasa de letalidad 
En la tabla 2 se aprecia el p-valor de las pruebas para las variables letalidad y vacunación contra COVID-19 que es de 0,001 , es menor a $\alpha=0,1$, de esta manera se acepta $\mathrm{Ha}$, es decir la prueba de correlación de Pearson nos indica que existe relación entre la tasa de letalidad por COVID-19 y la vacunación contra el SARS-COV-2 en Colombia durante el período comprendido entre 17 de febrero a 4 de junio 2021.

Tabla 2 Correlations de Pearson

\begin{tabular}{|l|l|c|c|}
\hline & & Vacunación día & Fallecidos \\
\hline Vacunación día & Correlación de Pearson & 1 &,$- 322^{* *}$ \\
\hline & Sig. (bilateral) & &, 001 \\
\hline & N & 108 & 108 \\
\hline Letalidad & Correlación de Pearson &,$- 322^{* *}$ & 1 \\
\hline & Sig. (bilateral) &, 001 & \\
\hline & N & 108 & 108 \\
\hline
\end{tabular}

** . La correlación es significativa en el nivel 0,01 (bilateral).

En la figura 2 se aprecia el diagrama de correlación entre la tasa de letalidad por COVID-19 y la vacunación contra SARS-COV-2. El número de datos es igual a 108; $r=-0.322$; $p<0,001$. De esta manera según el valor $r=-0,322$, se evidencia una correlación negativa baja entre las variables.
La ecuación lineal está dada por: $\mathrm{Y}=1,18 \mathrm{E} 5 \mathrm{X}-1,66 \mathrm{E} 5$, lo que se convierte que las dos variables se correlacionan en sentido inverso, a valores altos de la vacunación por COVID-19, le suelen corresponder valores bajos en la tasa de letalidad por SARS-COV-2.

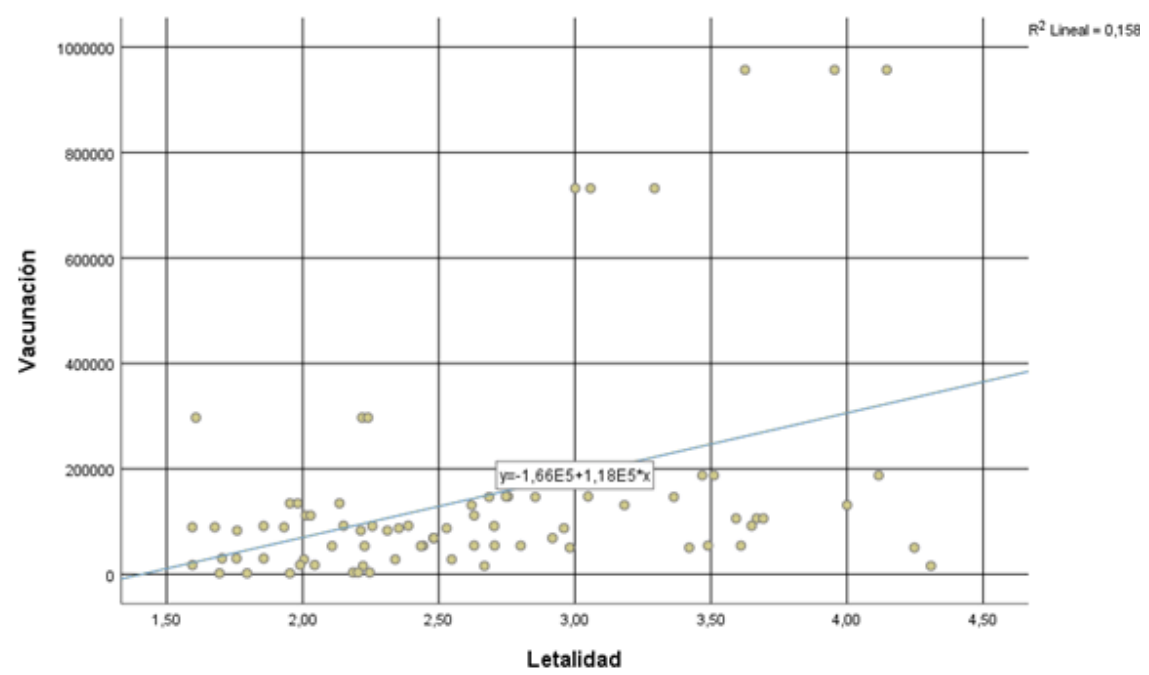

Figura 2 Diagrama de correlación por tasa de letalidad y vacunación.

\section{CONCLUSIONES}

Los niveles de correlación y determinación de Pearson a partir de los datos examinados, se convierte en que las dos variables se correlacionan en sentido inverso a valores altos de la vacunación por COVID-19, por lo tanto, le suelen corresponder valores bajos en la tasa de letalidad por SARS-
COV-2.Ahora bien, las vacunas contra COVID-19 tienen una alta efectividad para reducir los fallecimientos por SARS-COV-2, su efecto todavía no se evidencia en las cifras diarias de decesos, esto debido a las aglomeraciones por movilizaciones del paro de la población en Colombia y por 
la rapidez con la que se transmite el virus, el cual sobrepasa a la velocidad con las vacunas que generan inmunidad.

\section{REFERENCIAS}

1. Zhou P, Xing-Lou Y, Xian-Guang W, Ben H, Lei Z, Wei Z, Hao-Rui S, Yan Z, et al. A pneumonia outbreak associated with a new coronavirus of probable bat origin. Nature. 2020;579(7798):270-273. doi: 10.1038/ s41586-020-2012-7

2. Zhu N. Dingyu Z, Wenling W, Xingwang L, Bo Y, Jingdong S, Xiang Z, Baoying H, et al. A Novel Coronavirus from Patients with Pneumonia in China, 2019. N Engl J Med. 2020;382(8):727-733. doi: 10.1056/ NEJMoa2001017

3. Pan KY, Kok AA, Eikelenboom M, Horsfall M, Jörg F, et al. The mental health impact of the COVID-19 pandemic on people with and without depressive, anxiety, or obsessive-compulsive disorders: a longitudinal study of three Dutch case-control cohorts. Lancet Psychiatry. 2021;8(2):121-129. doi: 10.1016/S22150366(20)30491-0

4. Pierce M, Hope H, Ford T, Hatch S, Hotopf M, John A, et al. Mental health before and during the COVID-19 pandemic: a longitudinal probability sample survey of the UK population. Lancet Psychiatry. 2020;7(10):883-892. doi: 10.1016/S2215-0366(20)30308-4

5. Mofijur M, Rizwanul Fattah IM, Asraful Alam Md, Saiful Islam ABM, Chyuan Ong H, et al. Impact of COVID-19 on the social, economic, environmental and energy domains: Lessons learnt from a global pandemic. Sustain Prod Consum. 2021;26:343-359. doi: 10.1016/j.spc.2020.10.016

6. Baden LR, El Sahly HM, Essink B, Kotloff K, Frey S, et al. Efficacy and Safety of the mRNA-1273 SARS-CoV-2 Vaccine. N Engl J Med. 2021;384:403-416. doi: 10.1056/NEJMoa2035389

7. Voysey M, Costa Clemens SA, Madhi SA, Weckx LY, Folegatti PM, Aley PK, Angus B, et al. Safety and efficacy of the ChAdOxl nCoV19 vaccine (AZD1222) against SARS-CoV-2: an interim analysis of four randomised controlled trials in Brazil, South Africa, and the UK. Lancet. 2021;397(10269):99-111. doi: 10.1016/S0140-6736(20)32661-1

8. Polack FP, Stephen JT, Kitchin N, Absalon J, Gurtman A, Lockhart S, Perez JL, Gonzalo Pérez M. Safety and Efficacy of the BNT162b2 mRNA Covid-19 Vaccine. N Engl J Med. 2020;383(27):2603-2615. doi: 10.1056/NEJMoa2034577

9. Wang J, Peng Y, Xu H, Cui Z, Williams RO. The COVID-19 Vaccine Race: Challenges and Opportunities in Vaccine Formulation. AAPS PharmSciTech. 2020;21(6):225. doi: 10.1208/s12249-020-01744-7

10. Rawat K, Kumari P, Saha, L. COVID-19 vaccine: A recent update in pipeline vaccines, their design and development strategies. Eur J Pharmacol. 2021;892:173751. doi: 10.1016/j. ejphar.2020.173751

11. Abbasi K. Behavioural fatigue: a flawed idea central to a flawed pandemic response. BMJ. 2020;370:m3093. doi: 10.1136/bmj.m309312. Rypdal K, Bianchi FM, Rypdal, M. Intervention Fatigue is the Primary Cause of Strong Secondary Waves in the COVID-19 Pandemic. Int J Environ Res Public Health. 2020;17(24):9592. doi: 10.3390/ijerph 17249592
13. Priesemann V, Brinkmann MM, Ciesek S, Cuschieri S, Czypionka T, et al. Calling for pan-European commitment for rapid and sustained reduction in SARS-CoV-2 infections. Lancet. 2021;397(10269):9293. doi: 10.1016/S0140-6736(20)32625-8

14. Priesemann V, Balling R, Brinkmann MM, Ciesek S, Czypionka T, et al. An action plan for pan-European defence against new SARS-CoV-2 variants. Lancet. 2021;397(10273):469-470. doi: 10.1016/S0140-6736(21)00150-1

15. Davies NG, Abbott S, Barnard RC, Jarvis CI, Kucharski AJ, et al. Estimated transmissibility and impact of SARS-CoV-2 lineage B.1.1.7 in England. Science. 2021;372(6538):eabg3055. doi: 10.1126/science.abg 3055

16. Abbott S, Funk S, CMMID COVID-19 Working Group. Local area reproduction numbers and S-gene target failure [Internet]. Centre for Mathematical Modelling of Infectious Diseases 2021. [Citado 10 de junio de 2021]; Disponible en: https://cmmid.github.io/topics/ covid19/local-r-sgtf.html

17. Horby P, Huntley C, Davies N, Edmunds J, Ferguson N, Medley G, Semple C. NERVTAG paper on COVID-19 variant of concern B.1.1.7. Department of Health and Social Care. [Citado 10 de junio de 2021]; Disponible en: https://www.gov.uk/government/ publications/nervtag-paper-on-covid-19-variant-of-concern-b117

18. Bubar, KM, Reinholt K, Kissler SM, Lipsitch M, Cobey S et al. Model-informed COVID-19 vaccine prioritization strategies by age and serostatus. medRxiv. 2020;2020.09.08.20190629. doi: 10.1101/2020.09.08.20190629.

19. Ramos AM, Vela-Pérez M, Ferrández MR, Kubik AB, Ivorra B. Modeling the impact of SARS-CoV-2 variants and vaccines on the spread of COVID-19. Preprint in ResearchGate. [Citado 10 de junio de 2021]; Disponible en: https://doi.org/10.13140/RG.2.2.32580.24967/2

20. Suárez-Ibujes M. Coeficiente de correlación de Karl Pearson [Internet]. 2021. [citado 14 mayo 2021]; Disponible en: https:// www.monografias.com/trabajos85/coeficiente-correlacion-karlpearson/coeficiente-correlacion-karl-pearson.shtml

21. Moreno-Altamirano A. López-Moreno S, Corcho-Berdugo A. Principales medidas en epidemiología. Salud Pública de México. 2000;42(4):337-348.

22. Quintana-Salgado L. Medidas de frecuencia en epidemiología [Internet]. 2015. [Citado 10 de junio de 2021]; Disponible en: https://es.slideshare.net/lualberts20/medidas-de-frecuencia-enepidemiologa-2015

23. Díaz Pinzón JE. Estimación de las tasas de mortalidad y letalidad por COVID-19 en Colombia. Repert Med Cir. 2020;29(Núm. Supl.1):8993. https://doi.org/10.31260/RepertMedCir.01217372.1103

24. Díaz Pinzón, JE. Medidas de frecuencia por COVID-19 en Bogotá DC. Repert Med Cir. 2020;29(Núm. Supl.1):94-98. https://doi. org/10.31260/RepertMedCir.01217372.1110

25. Díaz Pinzón JE. Estimación de la prevalencia del COVID-19 en Colombia. Repert Med Cir. 2020;29(Núm. Supl.1):99-102. https:// doi.org/10.31260/RepertMedCir.01217372.1115

26. Ministerio de Salud y Protección Social. Plan Nacional de vacunación contra el COVID-19 [Internet]. 2021. [Citado $10 \mathrm{de}$ junio de 2021]; Disponible en: https://www.minsalud.gov.co/ salud/publica/Vacunacion/Paginas/Vacunacion-covid-19.aspx 